\title{
AUC from T1 to T2 Normalized by Body Mass Index
}

National Cancer Institute

\section{Source}

National Cancer Institute. AUC from T1 to T2 Normalized by Body Mass Index. NCI

Thesaurus. Code C92312.

The area under the curve (AUC) over the interval from T1 to T 2 divided by the body mass index. 